\title{
TRANSPORTATION INEQUALITIES UNDER UNIFORM METRIC FOR A STOCHASTIC HEAT EQUATION DRIVEN BY TIME-WHITE AND SPACE-COLORED NOISE
}

\author{
SHIJIE SHANG AND RAN WANG
}

\begin{abstract}
In this paper, we prove transportation inequalities on the space of continuous paths with respect to the uniform metric, for the law of solution to a stochastic heat equation defined on $[0, T] \times[0,1]^{d}$. This equation is driven by the Gaussian noise, white in time and colored in space. The proof is based on a new moment inequality under the uniform metric for the stochastic convolution with respect to the time-white and space-colored noise, which is of independent interest.
\end{abstract}

Keywords Stochastic heat equation, Transportation inequality, Girsanov transformation.

Mathematics Subject Classification (2000) 60E15; 60H15.

\section{INTRODUCTION}

The purpose of this paper is to study Talagrand's $T_{2}$-transportation inequality for the following $d$-dimensional spatial stochastic heat equation on $[0,1]^{d}$,

$$
\left\{\begin{array}{l}
\frac{\partial}{\partial t} u(t, x)=\Delta u(t, x)+\sigma(u(t, x)) \dot{F}(t, x)+b(u(t, x)), \quad t \geqslant 0, x \in(0,1)^{d}, \\
u(t, x)=0, \quad x \in \partial\left([0,1]^{d}\right), \\
u(0, x)=u_{0}(x), \quad x \in[0,1]^{d},
\end{array}\right.
$$

where $\Delta$ is the Laplacian operator on $(0,1)^{d}, \partial\left([0,1]^{d}\right)$ is the boundary of $[0,1]^{d}$, and $u_{0}$ is a continuous function on $[0,1]^{d}$ with $u_{0}(x)=0$ for any $x \in \partial\left([0,1]^{d}\right)$. Assume that the coefficients $\sigma$ and $b$ satisfy the following conditions:

(C1). $\sigma$ and $b$ are Lipschitzian, i.e., there exist some constants $L_{\sigma}, L_{b} \in[0, \infty)$ such that

$$
\left|\sigma\left(v_{1}\right)-\sigma\left(v_{2}\right)\right| \leqslant L_{\sigma}\left|v_{1}-v_{2}\right|, \quad\left|b\left(v_{1}\right)-b\left(v_{2}\right)\right| \leqslant L_{b}\left|v_{1}-v_{2}\right|, \quad \forall v_{1}, v_{2} \in \mathbb{R} .
$$

(C2). $\sigma$ is bounded, i.e., there exists a constant $K_{\sigma} \in(0, \infty)$ such that

$$
|\sigma(v)| \leqslant K_{\sigma}, \quad \forall v \in \mathbb{R} .
$$

Throughout this paper, we work on a filtered probability space $\left(\Omega, \mathcal{F},\left\{\mathcal{F}_{t}\right\}_{t \geqslant 0}, \mathbb{P}\right)$, where the filtration $\left\{\mathcal{F}_{t}\right\}_{t \geqslant 0}$ satisfies the usual conditions. The noise $F=\{F(\varphi), \varphi$ : $\left.\mathbb{R}^{d+1} \rightarrow \mathbb{R}\right\}$ is an $L^{2}(\Omega, \mathcal{F}, \mathbb{P})$-valued Gaussian process with mean zero and covariance functional given by

$$
J(\varphi, \psi):=\int_{\mathbb{R}_{+}} d s \int_{\mathbb{R}^{d}} d x \int_{\mathbb{R}^{d}} d y \varphi(s, x) f(x-y) \psi(s, y), \quad \varphi, \psi \in \mathcal{S}\left(\mathbb{R}^{d+1}\right),
$$

where $f: \mathbb{R}^{d} \rightarrow \mathbb{R}_{+}$is continuous on $\mathbb{R}^{d} \backslash\{0\}$, and $\mathcal{S}\left(\mathbb{R}^{d+1}\right)$ is the space of all Schwartz functions on $\mathbb{R}^{d+1}$, all of whose derivatives are rapidly decreasing. As a covariance functional of a Gaussian process, the functional $J(\cdot, \cdot)$ should be non-negative definite, 
this implies that $f$ is symmetric $\left(f(x)=f(-x)\right.$ for all $\left.x \in \mathbb{R}^{d}\right)$, and is equivalent to the existence of a non-negative tempered measure $\lambda$ on $\mathbb{R}^{d}$, whose Fourier transform is $f$. More precisely, the relationship between $\lambda$ and $f$ is that for all $\varphi \in \mathcal{S}\left(\mathbb{R}^{d}\right)$,

$$
\int_{\mathbb{R}^{d}} f(x) \varphi(x) d x=\int_{\mathbb{R}^{d}} \mathcal{F} \varphi(\xi) \lambda(d \xi),
$$

here $\mathcal{F} \varphi$ is the Fourier transform of $\varphi, \mathcal{F} \varphi(\xi):=\int_{\mathbb{R}^{d}} \exp (-2 i \pi \xi \cdot x) \varphi(x) d x$. See [7] for details.

In this paper, we assume the following hypothesis:

$\left(H_{\eta}\right)$. There exists a constant $\eta \in[0,1)$ satisfying that

$$
K_{\eta}:=\int_{\mathbb{R}^{d}} \frac{\lambda(d \xi)}{\left(1+|\xi|^{2}\right)^{\eta}}<+\infty .
$$

For instance, the tempered measure $\lambda$ associated with the function $f(x)=|x|^{-\kappa}, \kappa \in$ $(0,2 \wedge d)$, satisfies $(1.6)$.

As in R. Dalang [7], the Gaussian process $F$ can be extended to a worthy martingale measure, in the sense of J. Walsh [26], thus we can use the Walsh integral against $F$ to give the definition of mild solutions to (1.1) as follows. A continuous adapted random field $u=\left\{u(t, x),(t, x) \in \mathbb{R}_{+} \times[0,1]^{d}\right\}$ is called a mild solution of equation (1.1), if it satisfies

$$
\begin{aligned}
u(t, x)= & \int_{[0,1]^{d}} G_{t}(x, y) u_{0}(y) d y+\int_{0}^{t} \int_{[0,1]^{d}} G_{t-s}(x, y) \sigma(u(s, y)) F(d s, d y) \\
& +\int_{0}^{t} d s \int_{[0,1]^{d}} d y G_{t-s}(x, y) b(u(s, y)), \quad \mathbb{P}-\text { a.s. }, \quad x \in[0,1]^{d}, \quad t \geqslant 0,
\end{aligned}
$$

where $G_{t}(x, y)$ is the Green kernel associated with the heat equation on $[0,1]^{d}$ :

$$
\left\{\begin{array}{l}
\frac{\partial}{\partial t} G_{t}(x, y)=\Delta_{x} G_{t}(x, y), \quad t \geqslant 0, x, y \in(0,1)^{d} \\
G_{t}(x, y)=0, \quad x \in \partial\left([0,1]^{d}\right) \\
G_{0}(x, y)=\delta(x-y) .
\end{array}\right.
$$

The study of existence and uniqueness of solution to equation (1.7) on $\mathbb{R}^{d}$ has been studied by R. Dalang in [7]. Many other authors have also studied $d$-dimensional spatial stochastic heat equations, see $[12,14]$ and references therein. Under the assumption $(\mathrm{C} 1)$ and $\left(H_{\eta}\right)$ for $\eta \in[0,1)$, D. Márquez-Carreras and M. Sarrà [14] proved that equation (1.1) admits a unique solution.

In this paper, we shall study Talagrand's transportation inequality for solution of equation (1.1) under the uniform metric. Let us first recall the transportation inequality. Let $(E, d)$ be a metric space, and $\mathcal{M}(E)$ be the space of all probability measures on $E$. Given $\mu, \nu \in \mathcal{M}(E)$ and $p \geqslant 1$, the Wasserstein distance is defined by

$$
W_{p}(\mu, \nu):=\inf _{\pi}\left[\int_{E} \int_{E} d(x, y)^{p} \pi(d x, d y)\right]^{\frac{1}{p}}
$$


where the infimum is taken over all the probability measures $\pi$ on $E \times E$ with marginal distributions $\mu$ and $\nu$. The relative entropy of $\nu$ with respect to $\mu$ is defined as

$$
H(\nu \mid \mu):= \begin{cases}\int \log \frac{d \nu}{d \mu} d \nu, & \text { if } \nu \ll \mu ; \\ +\infty, & \text { otherwise }\end{cases}
$$

The probability measure $\mu$ satisfies the $T_{p}$-transportation inequality on $(E, d)$ if there exists a constant $C>0$ such that for any probability measure $\nu$ on $E$,

$$
W_{p}(\mu, \nu) \leqslant \sqrt{2 C H(\nu \mid \mu)} .
$$

As usual, we write $\mu \in \mathbf{T}_{\mathbf{p}}$ for this relation. The properties $\mathbf{T}_{\mathbf{1}}$ and $\mathbf{T}_{\mathbf{2}}$ are particularly interesting.

It is known that Talagrand's transportation inequality is closely related to the concentration of measure phenomenon, the log-Sobolev and Poincaré inequalities, see for instance monographs $[1,18,20,25]$. Recently, the problem of transportation inequalities to stochastic (partial) differential equations has been widely studied and is still a very active research area from both a theoretical and an applied point of view, for example see $[10,13,17]$ and references therein.

The work of M. Talagrand [24] on the Gaussian measure had been generalized by D. Feyel and A. S. Üstunel [11] to the framework of the abstract Wiener space. For stochastic differential equations (SDEs for short), by means of Girsanov transformation and the martingale representation theorem, the $\mathbf{T}_{2}$ w.r.t. the $L^{2}$ and the CameronMartin metrics were established by $\mathrm{H}$. Djellout et al. [9]; the $\mathbf{T}_{\mathbf{2}}$ w.r.t. the uniform metric was obtained by $\mathrm{L}$. Wu and Z. Zhang [28]. J. Bao et al. [2] established the $\mathbf{T}_{\mathbf{2}}$ w.r.t. both the uniform and the $L^{2}$ metrics on the path space for the segment processes associated with a class of neutral functional stochastic differential equations. B. Saussereau [22] studied the $\mathbf{T}_{\mathbf{2}}$ for SDEs driven by fractional Brownian motion, and S. Riedel [21] extended this result to SDEs driven by general Gaussian processes by using Lyons' rough paths theory.

For stochastic partial differential equations (SPDEs for short), L. Wu and Z. Zhang [29] studied the $\mathbf{T}_{\mathbf{2}}$ w.r.t. the $L^{2}$ metric by Galerkin approximations. B. Boufoussi and S. Hajji [4] obtained the $\mathbf{T}_{\mathbf{2}}$ w.r.t. the $L^{2}$ metric for stochastic heat equations driven by space-time white noise by Girsanov transformation, while D. Khoshnevisan and A. Sarantsev [16] studied this problem for more general SPDEs. T. Zhang and the first named author [23] established the $\mathbf{T}_{\mathbf{2}}$ w.r.t. the uniform metric for the stochastic heat equation driven by multiplicative space-time white noise. The above results are all forced on the SPDEs with deterministic initial values. Recently, F.-Y. Wang and T. Zhang [27] studied the transportation inequalities for SPDEs with random initial values.

The aim of this paper is to prove that under the uniform metric, the $\mathbf{T}_{\mathbf{2}}$ holds for stochastic heat equations driven by multiplicative time-white and space-colored noise. Our new contribution is the $p$ th-moment inequality under the uniform metric for the stochastic convolution with respect to the time-white and space-colored noise, which is of independent interest.

The rest of the paper is organized as follows. In Section 2, we first recall some facts about the stochastic integrals with respect to the time-white and space-colored noise, and establish the pth-moment inequality under the uniform metric for the stochastic 
convolution with respect to the time-white and space-colored noise. In Section 3, we prove the $\mathbf{T}_{\mathbf{2}}$ for the law of the solution to equation (1.1).

\section{Stochastic integrals And moment estimates for the stochastic EVOLUTION}

2.1. Stochastic integrals with respect to time-white and space-colored noise. Recall (1.5). Denote by $\mathcal{H}$ the Hilbert space obtained by the completion of $\mathcal{S}\left(\mathbb{R}^{d}\right)$ with respect to the inner product

$$
\begin{aligned}
\langle\phi, \psi\rangle_{\mathcal{H}} & :=\int_{\mathbb{R}^{d}} d x \int_{\mathbb{R}^{d}} d y \phi(x) \psi(y) f(x-y) \\
& =\int_{\mathbb{R}^{d}} \lambda(d \xi) \mathcal{F} \phi(\xi) \overline{\mathcal{F} \psi}(\xi), \quad \phi, \psi \in \mathcal{S}\left(\mathbb{R}^{d}\right),
\end{aligned}
$$

here $\bar{z}$ is the conjugate of the complex number $z$. The norm induced by $\langle\cdot, \cdot\rangle_{\mathcal{H}}$ is denoted by $\|\cdot\|_{\mathcal{H}}$.

Recall that the predictable $\sigma$-field on $\Omega \times[0, T]$ is generated by the sets $\{(s, t] \times A ; A \in$ $\left.\mathcal{F}_{s}, 0 \leqslant s<t \leqslant T\right\}$. There are two ways to define the stochastic integral against time-white and space-colored noise, see [8] for details.

On the one hand, as in R. Dalang [7], the Gaussian process $F$ can be extended to a worthy martingale measure in the sense of J. Walsh [26]. By using the approximation technique, for any $\mathcal{H}$-valued predictable process $g \in L^{2}(\Omega \times[0, T] ; \mathcal{H})$, the stochastic integral

$$
\int_{0}^{T} \int_{\mathbb{R}^{d}} g(s, x) F(d s, d x)
$$

is well-defined, see [7]. Furthermore, the above Walsh integral can be defined for $g \in$ $L^{2}([0, T] ; \mathcal{H}), \mathbb{P}$-a.s., by using localization techniques.

On the other hand, for any orthonormal basis $\left\{e_{k}\right\}_{k \geqslant 1}$ of the Hilbert space $\mathcal{H}$, the family of processes

$$
\left\{B_{t}^{k}:=\int_{0}^{t} \int_{\mathbb{R}^{d}} e_{k}(y) F(d s, d y), k \geqslant 1\right\}
$$

are a sequence of independent standard Wiener processes and the process $B_{t}:=\sum_{k \geqslant 1} B_{t}^{k} e_{k}$ is a cylindrical Brownian motion on $\mathcal{H}$. It is well-known that (see [6] or [8]) for any $\mathcal{H}$ valued predictable process $g \in L^{2}(\Omega \times[0, T] ; \mathcal{H})$, we can define the stochastic integral with respect to the cylindrical Wiener process $B$ as follows:

$$
\int_{0}^{T} g(s) d B_{s}:=\sum_{k \geqslant 1} \int_{0}^{T}\left\langle g(s), e_{k}\right\rangle_{\mathcal{H}} d B_{s}^{k} .
$$

Note that the above series converges in $L^{2}(\Omega, \mathcal{F}, \mathbb{P})$ and the sum does not depend on the selected orthonormal basis. Moreover, each summand, in the above series, is a classical Itô integral with respect to a standard Brownian motion. 
By [8, Proposition 2.6], we know that for any $\mathcal{H}$-valued predictable process $g \in L^{2}(\Omega \times$ $[0, T] ; \mathcal{H})$,

$$
\int_{0}^{T} \int_{\mathbb{R}^{d}} g(s, x) F(d s, d x)=\int_{0}^{T} g(s) d B_{s}
$$

2.2. Moment estimates for the stochastic convolution under the uniform metric. In this part, we will establish some moment estimates for the stochastic convolution driven by the time-white and space-colored noise. This part is inspired by [23] in the case of the space-time white noise.

For any random variable $\xi \in L^{p}(\Omega)$, let $\|\xi\|_{L^{p}(\Omega)}:=\left(\mathbb{E}|\xi|^{p}\right)^{\frac{1}{p}}$.

Proposition 2.1. Let $\left\{\sigma(s, y),(s, y) \in[0, T] \times[0,1]^{d}\right\}$ be a predictable random field and $\left\{p_{s}(x, y),(s, x, y) \in[0, T] \times[0,1]^{d} \times[0,1]^{d}\right\}$ be a deterministic function such that the following stochastic integral is well-defined. Then for any $t \in[0, T], x \in[0,1]^{d}$ and $p \geqslant 2$,

$$
\begin{aligned}
& \mathbb{E}\left[\left|\int_{0}^{t} \int_{[0,1]^{d}} p_{t-s}(x, y) \sigma(s, y) F(d s, d y)\right|^{p}\right] \\
\leqslant & (4 p)^{\frac{p}{2}}\left(\int_{0}^{t}\left\|p_{t-s}(x, \cdot)\right\| \sigma(s, \cdot)\left\|_{L^{p}(\Omega)}\right\|_{\mathcal{H}}^{2} d s\right)^{\frac{p}{2}} .
\end{aligned}
$$

Proof. By Burkholder's inequality (see [15, Proposition 4.4] and [7, Page 14]), we have

$$
\begin{aligned}
& \mathbb{E}\left|\int_{0}^{t} \int_{[0,1]^{d}} p_{t-s}(x, y) \sigma(s, y) F(d s, d y)\right|^{p} \\
\leqslant & (4 p)^{\frac{p}{2}} \mathbb{E}\left|\int_{0}^{t} d s \int_{[0,1]^{d}} d y \int_{[0,1]^{d}} d z p_{t-s}(x, y) p_{t-s}(x, z) f(y-z) \sigma(s, y) \sigma(s, z)\right|^{p / 2} .
\end{aligned}
$$

Taking $\frac{2}{p}$-th power on both sides of the above inequality and using Hölder's inequality, we get

$$
\begin{aligned}
& \left\|\int_{0}^{t} \int_{[0,1]^{d}} p_{t-s}(x, y) \sigma(s, y) F(d s, d y)\right\|_{L^{p}(\Omega)}^{2} \\
\leqslant & 4 p \int_{0}^{t} d s \int_{[0,1]^{d}} d y \int_{[0,1]^{d}} d z p_{t-s}(x, y) p_{t-s}(x, z) f(y-z) \sigma(s, y) \sigma(s, z) \|_{L^{\frac{p}{2}}(\Omega)} \\
\leqslant & 4 p \int_{0}^{t} d s \int_{[0,1]^{d}} d y \int_{[0,1]^{d}} d z p_{t-s}(x, y) p_{t-s}(x, z) f(y-z)\|\sigma(s, y) \sigma(s, z)\|_{L^{\frac{p}{2}}(\Omega)} \\
\leqslant & 4 p \int_{0}^{t} d s \int_{[0,1]^{d}} d y \int_{[0,1]^{d}} d z p_{t-s}(x, y) p_{t-s}(x, z) f(y-z)\|\sigma(s, y)\|_{L^{p}(\Omega)}\|\sigma(s, z)\|_{L^{p}(\Omega)} \\
= & 4 p \int_{0}^{t}\left\|p_{t-s}(x, \cdot)\right\| \sigma(s, \cdot)\left\|_{L^{p}(\Omega)}\right\|_{\mathcal{H}}^{2} d s .
\end{aligned}
$$

Then we take $\frac{p}{2}$-th power on both sides of the above inequality to obtain (2.14). The proof is complete. 
Proposition 2.2. Suppose hypothesis $\left(H_{\eta}\right)$ holds. Let $\left\{\sigma(s, y),(s, y) \in \mathbb{R}_{+} \times[0,1]^{d}\right\}$ be a random field such that the stochastic integral against the time-white and space-colored noise $F$ in (2.17) is well-defined. Then for any $T>0$ and $p>\frac{4+d}{1-\eta}$, there exists a constant $C_{T, p, \eta}>0$ such that

$$
\begin{aligned}
& \mathbb{E}\left[\sup _{(t, x) \in[0, T] \times[0,1]^{d}}\left|\int_{0}^{t} \int_{[0,1]^{d}} G_{t-s}(x, y) \sigma(s, y) F(d s, d y)\right|^{p}\right] \\
\leqslant & C_{T, p, \eta} \int_{0}^{T} \sup _{y \in[0,1]^{d}} \mathbb{E}|\sigma(s, y)|^{p} d s .
\end{aligned}
$$

Proof. The proof is based on the factorization method, which is inspired by [23]. We shall give its proof for the completeness. Obviously, we can assume that the right hand side of (2.17) is finite. Choose $\alpha$ such that $\frac{d+2}{2 p}<\alpha<\frac{1}{2}-\frac{1}{p}-\frac{\eta}{2}$. This is possible because $p>\frac{4+d}{1-\eta}$.

Let

$$
\begin{aligned}
\left(J_{\alpha} \sigma\right)(s, y) & :=\int_{0}^{s} \int_{[0,1]^{d}}(s-r)^{-\alpha} G_{s-r}(y, z) \sigma(r, z) F(d r, d z), \\
\left(J^{\alpha-1} f\right)(t, x) & :=\frac{\sin (\pi \alpha)}{\pi} \int_{0}^{t} \int_{[0,1]^{d}}(t-s)^{\alpha-1} G_{t-s}(x, y) f(s, y) d s d y .
\end{aligned}
$$

By the stochastic Fubini theorem, for any $(t, x) \in \mathbb{R}_{+} \times[0,1]^{d}$,

$$
\int_{0}^{t} \int_{[0,1]^{d}} G_{t-s}(x, y) \sigma(s, y) F(d s, d y)=J^{\alpha-1}\left(J_{\alpha} \sigma\right)(t, x), \quad \mathbb{P} \text {-a.s.. }
$$

Therefore

$$
\begin{aligned}
& \sup _{(t, x) \in[0, T] \times[0,1]^{d}}\left|\int_{0}^{t} \int_{[0,1]^{d}} G_{t-s}(x, y) \sigma(s, y) F(d s, d y)\right| \\
= & \sup _{(t, x) \in[0, T] \times[0,1]^{d}}\left|J^{\alpha-1}\left(J_{\alpha} \sigma\right)(t, x)\right|, \quad \mathbb{P} \text {-a.s.. }
\end{aligned}
$$

By Höler's inequality, (2.21) and (4.52) in Appendix, we have

$$
\begin{aligned}
& \mathbb{E} \sup _{(t, x) \in[0, T] \times[0,1]^{d}}\left|\int_{0}^{t} \int_{[0,1]^{d}} G_{t-s}(x, y) \sigma(s, y) F(d s, d y)\right|^{p} \\
= & \mathbb{E} \sup _{(t, x) \in[0, T] \times[0,1]^{d}}\left|\frac{\sin (\pi \alpha)}{\pi} \int_{0}^{t} \int_{[0,1]^{d}}(t-s)^{\alpha-1} G_{t-s}(x, y) J_{\alpha} \sigma(s, y) d s d y\right|^{p} \\
\leqslant & \left|\frac{\sin (\pi \alpha)}{\pi}\right|^{p} \mathbb{E} \sup _{(t, x) \in[0, T] \times[0,1]^{d}}\left\{\int_{0}^{t}(t-s)^{\alpha-1} \times\left(\int_{[0,1]^{d}} G_{t-s}(x, y)\left|J_{\alpha} \sigma(s, y)\right| d y\right) d s\right\}^{p} \\
\leqslant & \left|\frac{\sin (\pi \alpha)}{\pi}\right|^{p} \mathbb{E} \sup _{(t, x) \in[0, T] \times[0,1]^{d}}\left\{\int_{0}^{t}(t-s)^{\alpha-1} \times\left(\int_{[0,1]^{d}} G_{t-s}(x, y)\left|J_{\alpha} \sigma(s, y)\right|^{\frac{p}{2}} d y\right)^{\frac{2}{p}} d s\right\}^{p} \\
\leqslant & \left|\frac{\sin (\pi \alpha)}{\pi}\right|^{p} \sup _{(t, x) \in[0, T] \times[0,1]^{d}}\left\{\int_{0}^{t}(t-s)^{\alpha-1}\right.
\end{aligned}
$$




$$
\begin{aligned}
& \left.\times\left(\int_{[0,1]^{d}} G_{t-s}(x, y)^{2} d y\right)^{\frac{1}{p}}\left(\int_{[0,1]^{d}}\left|J_{\alpha} \sigma(s, y)\right|^{p} d y\right)^{\frac{1}{p}} d s\right\}^{p} \\
\leqslant & \left|\frac{\sin (\pi \alpha)}{\pi}\right|^{p}(4 \pi)^{-\frac{d}{2}} \mathbb{E} \sup _{t \in[0, T]}\left\{\int_{0}^{t}(t-s)^{\alpha-1-\frac{d}{2 p}}\left(\int_{[0,1]^{d}}\left|J_{\alpha} \sigma(s, y)\right|^{p} d y\right)^{\frac{1}{p}} d s\right\}^{p} \\
\leqslant & \left|\frac{\sin (\pi \alpha)}{\pi}\right|^{p}(4 \pi)^{-\frac{d}{2}} \mathbb{E} \sup _{t \in[0, T]}\left[\left(\int_{0}^{t}(t-s)^{\left(\alpha-1-\frac{d}{2 p}\right) \frac{p}{p-1}} d s\right)^{p-1} \times\left(\int_{0}^{t} \int_{[0,1]^{d}}\left|J_{\alpha} \sigma(s, y)\right|^{p} d y d s\right)\right] \\
\leqslant & \left|\frac{\sin (\pi \alpha)}{\pi}\right|^{p}(4 \pi)^{-\frac{d}{2}} \times\left(\int_{0}^{T} s^{\left(\alpha-1-\frac{d}{2 p}\right) \frac{p}{p-1}} d s\right)^{p-1} \times \int_{0}^{T} \int_{[0,1]^{d}} \mathbb{E}\left|J_{\alpha} \sigma(s, y)\right|^{p} d y d s \\
\leqslant & C_{T, p, \alpha}^{\prime} \sup _{(s, y) \in[0, T] \times[0,1]^{d}} \mathbb{E}\left|\int_{0}^{s} \int_{[0,1]^{d}}(s-r)^{-\alpha} G_{s-r}(y, z) \sigma(r, z) F(d r, d z)\right|^{p},
\end{aligned}
$$

where we have used the condition $\alpha>\frac{d}{2 p}+\frac{1}{p}$, so that

$$
\begin{aligned}
C_{T, p, \alpha}^{\prime} & =\left|\frac{\sin (\pi \alpha)}{\pi}\right|^{p}(4 \pi)^{-\frac{d}{2}} \times\left(\int_{0}^{T} s^{\left(\alpha-1-\frac{d}{2 p}\right) \frac{p}{p-1}} d s\right)^{p-1} \times T \\
& =\left|\frac{\sin (\pi \alpha)}{\pi}\right|^{p}(4 \pi)^{-\frac{d}{2}}\left(\frac{p-1}{\alpha p-1-\frac{d}{2}}\right)^{p-1} T^{\alpha p-\frac{d}{2}}<\infty .
\end{aligned}
$$

By Hölder's inequality, (2.14) and Lemma 4.1 in Appendix, we have

$$
\begin{aligned}
& \mathbb{E}\left|\int_{0}^{s} \int_{[0,1]^{d}}(s-r)^{-\alpha} G_{s-r}(y, z) \sigma(r, z) F(d r, d z)\right|^{p} \\
\leqslant & (4 p)^{\frac{p}{2}}\left(\int_{0}^{s}(s-r)^{-2 \alpha} \cdot\left\|G_{s-r}\right\|_{\mathcal{H}}^{2} \cdot \sup _{z \in[0,1]^{d}}\|\sigma(r, z)\|_{L^{p}(\Omega)}^{2} d r\right)^{\frac{p}{2}} \\
\leqslant & (4 p)^{\frac{p}{2}}\left(\int_{0}^{s}\left[(s-r)^{-2 \alpha}\left\|G_{s-r}\right\|_{\mathcal{H}}^{2}\right]^{\frac{p}{p-2}} d r\right)^{\frac{p-2}{2}} \times\left(\int_{0}^{s} \sup _{z \in[0,1]^{d}}\|\sigma(r, z)\|_{L^{p}(\Omega)}^{p} d r\right) \\
\leqslant & (4 p)^{\frac{p}{2}}\left(\int_{0}^{s}\left[(s-r)^{-2 \alpha} \times K_{\eta}\left(1 \vee\left(\frac{\eta}{8 \pi^{2}}\right)^{\eta}(s-r)^{-\eta}\right)\right]^{\frac{p}{p-2}} d r\right)^{\frac{p-2}{2}} \\
& \times\left(\int_{0}^{s} \sup _{z \in[0,1]^{d}}\|\sigma(r, z)\|_{L^{p}(\Omega)}^{p} d r\right) \\
\leqslant & C_{T, p, \alpha, \eta}^{\prime \prime} \int_{0}^{s} \sup _{z \in[0,1]^{d}} \mathbb{E}|\sigma(r, z)|^{p} d r,
\end{aligned}
$$

where we have used the condition $\alpha<\frac{1}{2}-\frac{1}{p}-\frac{\eta}{2}$, so that

$$
\begin{aligned}
& C_{T, p, \alpha, \eta}^{\prime \prime} \\
= & \left(4 p K_{\eta}\right)^{\frac{p}{2}} \sup _{s \in[0, T]}\left(\int_{0}^{s}(s-r)^{-\frac{2 \alpha p}{p-2}}\left[1 \vee\left(\frac{\eta}{8 \pi^{2}}\right)^{\eta}(s-r)^{-\frac{\eta p}{p-2}}\right] d r\right)^{\frac{p-2}{2}}
\end{aligned}
$$




$$
\begin{aligned}
& \leqslant\left(4 p K_{\eta}\right)^{\frac{p}{2}}\left(\frac{p-2}{p-2-2 \alpha p} T^{\frac{p-2-2 \alpha p}{p-2}}+\left(\frac{\eta}{8 \pi^{2}}\right)^{\eta} \frac{p-2}{p-2-2 \alpha p-\eta p} T^{\frac{p-2-2 \alpha p-\eta p}{p-2}}\right)^{\frac{p-2}{2}} \\
& \leqslant \frac{1}{4}\left(8 p K_{\eta}\right)^{\frac{p}{2}}\left[\left(\frac{p-2}{p-2-2 \alpha p}\right)^{\frac{p-2}{2}} T^{\frac{p}{2}-1-\alpha p}+\left(\frac{\eta}{8 \pi^{2}}\right)^{\frac{\eta(p-2)}{2}}\left(\frac{p-2}{p-2-2 \alpha p-\eta p}\right)^{\frac{p-2}{2}} T^{\frac{p}{2}-1-\alpha p-\frac{\eta p}{2}}\right] \\
& <\infty .
\end{aligned}
$$

Combining (2.22) with (2.24), we obtain

$$
\begin{aligned}
& \mathbb{E} \sup _{(t, x) \in[0, T] \times[0,1]^{d}}\left|\int_{0}^{t} \int_{[0,1]^{d}} G_{t-s}(x, y) \sigma(s, y) F(d s, d y)\right|^{p} \\
\leqslant & C_{T, p, \eta} \int_{0}^{T} \sup _{z \in[0,1]^{d}} \mathbb{E}|\sigma(s, z)|^{p} d s,
\end{aligned}
$$

where

$$
C_{T, p, \eta}=\min _{\frac{d+2}{2 p}<\alpha<\frac{1}{2}-\frac{1}{p}-\frac{\eta}{2}} C_{T, p, \alpha}^{\prime} \times C_{T, p, \alpha, \eta}^{\prime \prime}
$$

In view of (2.23) and (2.25), a straightforward calculation leads to

$$
\begin{aligned}
C_{T, p, \eta}<p^{\frac{p}{2}} & \times \frac{1}{4}\left(\frac{1}{4 \pi}\right)^{\frac{d}{2}}\left(\frac{\sqrt{8 K_{\eta}}}{\pi}\right)^{p} \\
\times & {\left[\max \left\{\left(\frac{3 p-4}{p-4-d}\right)^{\frac{3 p}{2}-2},\left(\frac{2(p-1)}{(1-\eta) p-4-d}\right)^{p-1}\left(\frac{p-2}{p \eta}\right)^{\frac{p}{2}-1}\right\} T^{\frac{p}{2}-1-\frac{d}{2}}\right.} \\
+ & \left.\left(\frac{\eta}{8 \pi^{2}}\right)^{\frac{\eta(p-2)}{2}}\left(\frac{3 p-4}{(1-\eta) p-4-d}\right)^{\frac{3 p}{2}-2} T^{\frac{(1-\eta) p}{2}-1-\frac{d}{2}}\right] .
\end{aligned}
$$

This completes the proof of the estimate (2.17).

Based on Proposition 2.2, we can obtain the following estimate by using the argument in the proof of [23, Proposition 3.4 ]. The proof is omitted here.

Proposition 2.3. Suppose hypothesis $\left(H_{\eta}\right)$ holds. Let $\left\{\sigma(s, y),(s, y) \in \mathbb{R}_{+} \times[0,1]^{d}\right\}$ be as in Proposition 2.2. Then for any $T>0, \varepsilon>0$ and $0<p \leqslant \frac{4+d}{1-\eta}$, there exists a constant $C_{T, p, \eta, \varepsilon}$ such that

$$
\begin{aligned}
& \mathbb{E}\left[\sup _{(t, x) \in[0, T] \times[0,1]^{d}}\left|\int_{0}^{t} \int_{[0,1]^{d}} G_{t-s}(x, y) \sigma(s, y) F(d s, d y)\right|^{p}\right] \\
\leqslant & \varepsilon \mathbb{E}\left[\sup _{(s, y) \in[0, T] \times[0,1]^{d}}|\sigma(s, y)|^{p}\right]+C_{T, p, \eta, \varepsilon} \mathbb{E} \int_{0}^{T} \sup _{y \in[0,1]^{d}}|\sigma(s, y)|^{p} d s,
\end{aligned}
$$

where

$$
C_{T, p, \eta, \varepsilon}=\inf _{q>\frac{4+d}{1-\eta}}\left\{\left(1+\frac{q C_{T, p, \eta}}{q-p}\right) p q^{-\frac{q}{p}}\left(q-p+q C_{T, p, \eta}\right)^{\frac{q}{p}-1} \varepsilon^{\frac{q}{p}-1}\right\},
$$

and the constant $C_{T, p, \eta}$ is bounded by the right hand side of (2.28). 


\section{Transportation InEQUALity}

3.1. The main results. Let $E:=\mathcal{C}_{0}\left([0,1]^{d} ; \mathbb{R}\right)$ be the space of all continuous functions $f$ from $[0,1]^{d}$ to $\mathbb{R}$ satisfying $f(x)=0$ for all $x \in \partial\left([0,1]^{d}\right)$, endowed with the uniform metric

$$
d_{E, \infty}\left(f_{1}, f_{2}\right):=\sup _{x \in[0,1]^{d}}\left|f_{1}(x)-f_{2}(x)\right|, \quad f_{1}, f_{2} \in E,
$$

and let $E_{T}:=\mathcal{C}([0, T] ; E)$ be the space of all continuous functions from $[0, T]$ to $E$, endowed with the uniform metric

$$
d_{E_{T}, \infty}\left(u_{1}, u_{2}\right):=\sup _{(t, x) \in \times[0, T] \times[0,1]^{d}}\left|u_{1}(t, x)-u_{2}(t, x)\right|, \quad u_{1}, u_{2} \in E_{T} .
$$

Let $\left\{u(t, x),(t, x) \in[0, T] \times[0,1]^{d}\right\}$ be the unique solution of the equation (1.1). For any $\mu \in \mathcal{M}(E)$, let $P^{\mu}$ be the distribution of the solution $\left\{u(t, x),(t, x) \in[0, T] \times[0,1]^{d}\right\}$ on $E_{T}$ such that the law of $u_{0}$ is $\mu$. Particularly, if $\mu=\delta_{u_{0}}$ for some $u_{0} \in E$, we write $P^{u_{0}}:=P^{\delta_{\mu_{0}}}$ for short.

Here are the main results of this section.

Theorem 3.1. Under (C1), (C2) and $\left(H_{\eta}\right)$, for any deterministic initial value $u_{0} \in E$. the law $P^{u_{0}}$ satisfies the $T_{2}$-transportation inequality on the space $E_{T}$ with respect to the uniform metric.

Applying Proposition 2.3 and Theorem 3.1 and using the same approach in the proof of [27, Theorem 3.1], we can get the following transportation inequality for the stochastic heat equation with random initial values, whose proof is omitted here.

Corollary 3.2. Under (C1), (C2) and $\left(H_{\eta}\right)$, and $\mu \in \mathcal{M}(E)$. Then

$$
W_{2}^{2}\left(Q, P^{\mu}\right) \leqslant 2 C H\left(Q \mid P^{\mu}\right), \quad \forall Q \in \mathcal{M}\left(E_{T}\right)
$$

holds for some constant $C>0$ if and only if

$$
W_{2}^{2}(\nu, \mu) \leqslant 2 c H(\nu \mid \mu), \quad \forall \nu \in \mathcal{M}(E)
$$

holds for some constant $c>0$.

3.2. The proof of Theorem 3.1. We will apply the Girsanov theorem to prove Theorem 3.1. To do this, we need the following lemma describing all probability measures which are absolutely continuous with respect to $P^{u_{0}}$. It is analogous to [9, Theorem 5.6] in the setting of finite-dimensional Brownian motion and [16, Lemma 3.1] in the setting of space-time white noise. For the completeness, we give its proof here.

Lemma 3.1. Let $Q$ be a probability measure on $E_{T}$ such that $Q \ll P^{u_{0}}$. Define a new probability measure $\mathbb{Q}$ on the probability space $(\Omega, \mathcal{F}, \mathbb{P})$ by

$$
d \mathbb{Q}:=\frac{d Q}{d P^{u_{0}}}(u) d \mathbb{P}
$$

Then there exists an $\mathcal{H}$-valued predictable process $h=\{h(s), s \in[0, T]\}$ such that

$$
\int_{0}^{T}\|h(s)\|_{\mathcal{H}}^{2} d s<\infty, \quad \mathbb{Q}-\text { a.s. }
$$


and the process

$$
\widetilde{B}_{t}:=B_{t}-\int_{0}^{t} h(s) d s, \quad t \in[0, T],
$$

is a cylindrical Wiener process on $\mathcal{H}$ under $\mathbb{Q}$. Furthermore,

$$
H\left(Q \mid P^{u_{0}}\right)=\frac{1}{2} \mathbb{E}^{\mathbb{Q}} \int_{0}^{T}\|h(s)\|_{\mathcal{H}}^{2} d s,
$$

where $\mathbb{E}^{\mathbb{Q}}$ denotes the expectation under the probability measure $\mathbb{Q}$.

Proof. The proof is adapted from [9, Theorem 5.6]. Let

$$
M_{t}:=\left.\frac{d \mathbb{Q}}{d \mathbb{P}}\right|_{\mathcal{F}_{t}}, \quad t \in[0, T]
$$

Then $\left(M_{t}\right)_{t \in[0, T]}$ is a nonnegative $\mathbb{P}$-martingale. Let $\tau:=\inf \{t \geqslant 0 ; M(t)=0\} \wedge T$ with the convention inf $\varnothing:=\infty$. Then $\mathbb{Q}(\tau=T)=1$, and the martingale $M$ can be represented as the stochastic exponential of another continuous local martingale $L$ :

$$
M(t)=\exp \left(L(t)-\frac{1}{2}[L]_{t}\right), \quad t<\tau,
$$

where $L(t)=\int_{0}^{t} \frac{d M(s)}{M(s)}$ for $t<\tau$. By the martingale representation theorem (e.g., [5, Theorem 2.3]), we know that there is a predictable process $h=\{h(t) ; t \in[0, \tau)\}$ valued in $\mathcal{H}$ such that

$$
\int_{0}^{t}\|h(s)\|_{\mathcal{H}}^{2} d s<+\infty, \quad t<\tau, \quad \mathbb{P}-\text { a.s. },
$$

and

$$
L(t)=\int_{0}^{t} h(s) d B_{s}=\sum_{k=1}^{\infty} \int_{0}^{t}\left\langle h(s), e_{k}\right\rangle_{\mathcal{H}} d B_{s}^{k}, \quad t<\tau,
$$

where the above integral is defined as in (2.12). By the Girsanov theorem (e.g., [5, Theorem 2.2] or [6, Theorem 10.14]), we know that

$$
\widetilde{B}_{t}:=B_{t}-\int_{0}^{t} h(s) d s, \quad t \in[0, T]
$$

is a cylindrical Wiener process on $\mathcal{H}$ under $\mathbb{Q}$.

Let $\tau_{n}:=\inf \left\{t \in[0, \tau) ;[L]_{t}=n\right\} \wedge \tau$ with the same convention that inf $\varnothing:=\infty$. Then $\tau_{n} \uparrow \tau, \mathbb{P}$-a.s., and by the martingale convergence theorem, we have

$$
\begin{aligned}
H\left(Q \mid P^{u_{0}}\right) & =\mathbb{E}^{\mathbb{P}} M_{T} \log M_{T}=\lim _{n \rightarrow \infty} \mathbb{E}^{\mathbb{P}} M_{T \wedge \tau_{n}} \log M_{T \wedge \tau_{n}}=\lim _{n \rightarrow \infty} \mathbb{E}^{\mathbb{Q}} \log M_{T \wedge \tau_{n}} \\
& =\lim _{n \rightarrow \infty} \mathbb{E}^{\mathbb{Q}}\left(L\left(T \wedge \tau_{n}\right)-\frac{1}{2}[L]_{T \wedge \tau_{n}}\right) .
\end{aligned}
$$

By Girsanov formula, $\left\{L\left(t \wedge \tau_{n}\right)-[L]_{t \wedge \tau_{n}}, t \in[0, T]\right\}$ is a $\mathbb{Q}$-local martingale, then a true martingale since its quadratic variation process under $\mathbb{Q}$, being again $\left\{[L]_{t \wedge \tau_{n}}, t \in[0, T]\right\}$, is bounded by $n$. Consequently, $\mathbb{E}^{\mathbb{Q}}\left(L\left(T \wedge \tau_{n}\right)-[L]_{T \wedge \tau_{n}}\right)=0$. Substituting it into the proceeding equality and by the monotone convergence, we have

$$
H\left(Q \mid P^{u_{0}}\right)=\frac{1}{2} \lim _{n \rightarrow \infty} \mathbb{E}^{\mathbb{Q}}\left([L]_{T \wedge \tau_{n}}\right)=\frac{1}{2} \mathbb{E}^{\mathbb{Q}}[L]_{T}=\frac{1}{2} \mathbb{E}^{\mathbb{Q}} \int_{0}^{T}\|h(s)\|_{\mathcal{H}}^{2} d s .
$$


The proof is complete.

Proof of Theorem 3.1. For any $Q \ll P^{u_{0}}$ such that $H\left(Q \mid P^{u_{0}}\right)<\infty$, let $\mathbb{Q}$ be defined as (3.33) and $h$ be the corresponding process appeared in Lemma 3.1. Then it is easy to see that the solution of equation (1.1) satisfies the following equation:

$$
\begin{aligned}
u(t, x)= & \int_{[0,1]^{d}} G_{t}(x, y) u_{0}(y) d y+\sum_{k \geqslant 1} \int_{0}^{t}\left\langle G_{t-s}(x, \cdot) \sigma(u(s, \cdot)), e_{k}\right\rangle_{\mathcal{H}} d \widetilde{B}_{s}^{k} \\
& +\int_{0}^{t} \int_{[0,1]^{d}} G_{t-s}(x, y) b(u(s, y)) d y d s \\
& +\sum_{k \geqslant 1} \int_{0}^{t}\left\langle G_{t-s}(x, \cdot) \sigma(u(s, \cdot)), e_{k}\right\rangle_{\mathcal{H}} \cdot\left\langle h(s), e_{k}\right\rangle_{\mathcal{H}} d s .
\end{aligned}
$$

Consider the solution of the following equation:

$$
\begin{aligned}
v(t, x)= & \int_{[0,1]^{d}} G_{t}(x, y) u_{0}(y) d y+\sum_{k \geqslant 1} \int_{0}^{t}\left\langle G_{t-s}(x, \cdot) \sigma(v(s, \cdot)), e_{k}\right\rangle_{\mathcal{H}} d \widetilde{B}_{s}^{k} \\
& +\int_{0}^{t} \int_{[0,1]^{d}} G_{t-s}(x, y) b(v(s, y)) d y d s .
\end{aligned}
$$

By Lemma 3.1, (2.12), and (2.13), it follows that under probability measure $\mathbb{Q}$, the law of $(v, u)$ forms a coupling of $(\mu, \nu)$. Therefore, by the definition of the Wasserstein distance, we have

$$
W_{2}^{2}\left(Q, P^{u_{0}}\right) \leqslant \mathbb{E}^{\mathbb{Q}}\left[\sup _{(t, x) \in[0, T] \times[0,1]^{d}}|u(t, x)-v(t, x)|^{2}\right] .
$$

In view of (3.36) and (3.40), to prove the $T_{2}$-transportation inequality, it is sufficient to show that

$$
\mathbb{E}^{\mathbb{Q}}\left[\sup _{(t, x) \in[0, T] \times[0,1]^{d}}|u(t, x)-v(t, x)|^{2}\right] \leqslant C \mathbb{E}^{\mathbb{Q}} \int_{0}^{T}\|h(s)\|_{\mathcal{H}}^{2} d s .
$$

for some constant $C$ independent of $v$. From (3.38) and (3.39), we have

$$
\begin{aligned}
u(t, x)-v(t, x)= & \sum_{k \geqslant 1} \int_{0}^{t}\left\langle G_{t-s}(x-\cdot)[\sigma(u(s, \cdot))-\sigma(v(s, \cdot))], e_{k}\right\rangle_{\mathcal{H}} d \widetilde{B}_{s}^{k} \\
& +\int_{0}^{t} \int_{[0,1]^{d}} G_{t-s}(x, y)[b(u(s, y))-b(v(s, y))] d y d s \\
& +\sum_{k \geqslant 1} \int_{0}^{t}\left\langle G_{t-s}(x, \cdot) \sigma(u(s, \cdot)), e_{k}\right\rangle_{\mathcal{H}} \cdot\left\langle h(s), e_{k}\right\rangle_{\mathcal{H}} d s .
\end{aligned}
$$

Thus,

$$
|u(t, x)-v(t, x)|^{2} \leqslant 3\left|\sum_{k \geqslant 1} \int_{0}^{t}\left\langle G_{t-s}(x, \cdot)[\sigma(u(s, \cdot))-\sigma(v(s, \cdot))], e_{k}\right\rangle_{\mathcal{H}} d \widetilde{B}_{s}^{k}\right|^{2}
$$




$$
\begin{aligned}
& +3\left|\int_{0}^{t} \int_{[0,1]^{d}} G_{t-s}(x, y)[b(u(s, y))-b(v(s, y))] d y d s\right|^{2} \\
& +3\left|\sum_{k \geqslant 1} \int_{0}^{t}\left\langle G_{t-s}(x, \cdot) \sigma(u(s, \cdot)), e_{k}\right\rangle_{\mathcal{H}} \cdot\left\langle h(s), e_{k}\right\rangle_{\mathcal{H}} d s\right|^{2} \\
= & : 3\left[\left|I_{1}(t, x)\right|^{2}+\left|I_{1}(t, x)\right|^{2}+\left|I_{3}(t, x)\right|^{2}\right] .
\end{aligned}
$$

By Proposition 2.3, we obtain that for any $\varepsilon>0$,

$$
\begin{aligned}
& \mathbb{E}^{\mathbb{Q}}\left[\sup _{(t, x) \in[0, T] \times[0,1]^{d}}\left|I_{1}(t, x)\right|^{2}\right] \\
\leqslant & \varepsilon \mathbb{E}^{\mathbb{Q}}\left[\sup _{(t, x) \in[0, T] \times[0,1]^{d}}|\sigma(u(t, x))-\sigma(v(t, x))|^{2}\right] \\
& +C_{T, 2, \eta, \varepsilon} \mathbb{E}^{\mathbb{Q}} \int_{0}^{T} \sup _{y \in[0,1]^{d}}|\sigma(u(s, y))-\sigma(v(s, y))|^{2} d s \\
\leqslant & L_{\sigma}^{2} \mathbb{E}^{\mathbb{Q}}\left[\sup _{(t, x) \in[0, T] \times[0,1]^{d}}|u(t, x)-v(t, x)|^{2}\right] \\
& +C_{T, 2, \eta, \varepsilon} L_{\sigma}^{2} \int_{0}^{T} \mathbb{E}_{(r, x) \in[0, s] \times[0,1]^{d}}^{\mathbb{Q}}|u(r, x)-v(r, x)|^{2} d s,
\end{aligned}
$$

where $C_{T, 2, \eta, \varepsilon}$ is the constant in (2.30) with $p=2$. By Cauchy-Schwarz's inequality, (4.51) in Appendix and the Lipschitz continuity of $b$, we obtain that

$$
\begin{aligned}
& \mathbb{E}^{\mathbb{Q}}\left[\sup _{(t, x) \in[0, T] \times[0,1]^{d}}\left|I_{2}(t, x)\right|^{2}\right] \\
\leqslant & \left(\sup _{(t, x) \in[0, T] \times[0,1]^{d}} \int_{0}^{t} \int_{[0,1]^{d}} G_{t-s}(x, y) d y d s\right) \\
& \times \mathbb{E}^{\mathbb{Q}}\left(\sup _{(t, x) \in[0, T] \times[0,1]^{d}} \int_{0}^{t} \int_{[0,1]^{d}} G_{t-s}(x, y)|b(u(s, y))-b(v(s, y))|^{2} d y d s\right) \\
\leqslant & T L_{b}^{2} \mathbb{E}^{\mathbb{Q}}\left(\sup _{(t, x) \in[0, T] \times[0,1]^{d}} \int_{0}^{t} \sup _{(r, y) \in[0, s] \times[0,1]^{d}}|u(r, y)-v(r, y)|^{2} \int_{[0,1]^{d}} G_{t-s}(x, y) d y d s\right) \\
\leqslant & T L_{b}^{2} \int_{0}^{T} \operatorname{Eup}_{(r, y) \in[0, s] \times[0,1]^{d}}^{\mathbb{Q}}|u(r, y)-v(r, y)|^{2} d s .
\end{aligned}
$$

For the third term, by assumption (C2) and Lemma 4.1 in Appendix, we have

$$
\begin{aligned}
& \mathbb{E}^{\mathbb{Q}}\left[\sup _{(t, x) \in[0, T] \times[0,1]^{d}}\left|I_{3}(t, x)\right|^{2}\right] \\
\leqslant & K_{\sigma}^{2}\left(\sup _{(t, x) \in[0, T] \times[0,1]^{d}} \int_{0}^{t}\left\|G_{t-s}(x, \cdot)\right\|_{\mathcal{H}}^{2} d s\right) \times \mathbb{E}^{\mathbb{Q}}\left(\int_{0}^{T}\|h(s)\|_{\mathcal{H}}^{2} d s\right)
\end{aligned}
$$




$$
\leqslant K_{\sigma}^{2} C_{G, T, \eta} \mathbb{E}^{\mathbb{Q}} \int_{0}^{T}\|h(s)\|_{\mathcal{H}}^{2} d s,
$$

where $C_{G, T, \eta}$ is the constant given in (4.55). Now, for every $T>0$, define

$$
Y(T):=\mathbb{E}^{\mathbb{Q}}\left[\sup _{(s, x) \in[0, T] \times[0,1]^{d}}|u(s, x)-v(s, x)|^{2}\right] .
$$

Recall that (see e.g. Theorem 3.2 in [14] or Proposition 3.2 in [19], combined with Garsia's lemma)

$$
\mathbb{E}^{\mathbb{Q}}\left[\sup _{(t, x) \in[0, T] \times[0,1]^{d}}|u(t, x)|^{2}\right]+\mathbb{E}^{\mathbb{Q}}\left[\sup _{(t, x) \in[0, T] \times[0,1]^{d}}|v(t, x)|^{2}\right]<\infty .
$$

Hence $Y(T)<\infty$ for any $T>0$. Putting (3.43)-(3.46) together, we have

$$
\begin{aligned}
Y(T) \leqslant & 3 \varepsilon L_{\sigma}^{2} Y(T)+3\left(C_{T, 2, \eta, \varepsilon} L_{\sigma}^{2}+T L_{b}^{2}\right) \int_{0}^{T} Y(s) d s \\
& +3 K_{\sigma}^{2} C_{G, T, \eta} \mathbb{E}^{\mathbb{Q}} \int_{0}^{T}\|h(s)\|_{\mathcal{H}}^{2} d s .
\end{aligned}
$$

Taking $\varepsilon=\varepsilon_{0}:=\frac{1}{6 L_{\sigma}^{2}}$ and subtracting $\frac{1}{2} Y(T)$ from both sides of the above inequality yield

$$
Y(T) \leqslant 6\left(C_{T, 2, \eta, \varepsilon_{0}} L_{\sigma}^{2}+T L_{b}^{2}\right) \int_{0}^{T} Y(s) d s+6 K_{\sigma}^{2} C_{G, T, \eta} \mathbb{E}^{\mathbb{Q}} \int_{0}^{T}\|h(s)\|_{\mathcal{H}}^{2} d s .
$$

Obviously, (3.49) still holds if we replace $T$ with any $t \in[0, T]$. Hence we can use Gronwall's inequality to obtain that

$$
Y(T) \leqslant 6 K_{\sigma}^{2} C_{G, T, \eta} e^{6\left(C_{T, 2, \eta, \varepsilon_{0}} L_{\sigma}^{2}+T L_{b}^{2}\right) T} \mathbb{E}^{\mathbb{Q}} \int_{0}^{T}\|h(s)\|_{\mathcal{H}}^{2} d s .
$$

This proves (3.41). The proof is complete.

\section{APPENDIX}

To make reading easier, we present here some results on the kernel $G$ associated with equation (1.8).

Let

$$
H_{t}(x):=\left(\frac{1}{4 \pi t}\right)^{\frac{d}{2}} \exp \left(-\frac{|x|^{2}}{4 t}\right), \quad x \in \mathbb{R}^{d}, t>0 .
$$

From Lemma 7 of [3], we have

$$
G_{t}(x, y) \leqslant H_{t}(x-y), \quad \forall t>0, x, y \in[0,1]^{d} .
$$

Hence, it is easy to see that

$$
\int_{[0,1]^{d}} G_{t}(x, y) d y<1
$$




$$
\int_{[0,1]^{d}} G_{t}(x, y)^{2} d y<\sup _{y \in[0,1]^{d}} G_{t}(x, y) \times \int_{[0,1]^{d}} G_{t}(x, y) d y<(4 \pi)^{-\frac{d}{2}} t^{-\frac{d}{2}} .
$$

Lemma 4.1. Suppose hypothesis $\left(H_{\eta}\right)$ holds. Then for any $t>0$ and $x \in[0,1]^{d}$,

$$
\left\|G_{t}(x, \cdot)\right\|_{\mathcal{H}}^{2} \leqslant K_{\eta}\left(1 \vee\left(\frac{\eta}{8 \pi^{2}}\right)^{\eta} t^{-\eta}\right),
$$

where $K_{\eta}$ is the constant in (1.6). Consequently, we have

$$
\int_{0}^{T}\left\|G_{t}(x, \cdot)\right\|_{\mathcal{H}}^{2} d t \leqslant C_{G, T, \eta}, \quad \forall x \in[0,1]^{d}
$$

where

$$
C_{G, T, \eta}:= \begin{cases}(1-\eta)^{-1} K_{\eta}\left(\frac{\eta}{8 \pi^{2}}\right)^{\eta} T^{1-\eta}, & \text { if } T \leqslant \frac{\eta}{8 \pi^{2}} \\ K_{\eta} T+\frac{\eta^{2}}{8 \pi^{2}(1-\eta)}, & \text { if } T>\frac{\eta}{8 \pi^{2}}\end{cases}
$$

Proof. By (2.10) and (4.50), we have for any $t>0$,

$$
\begin{aligned}
\left\|G_{t}(x, \cdot)\right\|_{\mathcal{H}}^{2} & =\int_{[0,1]^{d}} d y_{1} \int_{[0,1]^{d}} d y_{2} G_{t}\left(x, y_{1}\right) G_{t}\left(x, y_{2}\right) f\left(y_{1}-y_{2}\right) \\
& \leqslant \int_{\mathbb{R}^{d}} d y_{1} \int_{\mathbb{R}^{d}} d y_{2} H_{t}\left(x-y_{1}\right) H_{t}\left(x-y_{2}\right) f\left(y_{1}-y_{2}\right) \\
& =\int_{\mathbb{R}^{d}} d y_{1} \int_{\mathbb{R}^{d}} d y_{2} H_{t}\left(y_{1}\right) H_{t}\left(y_{2}\right) f\left(y_{1}-y_{2}\right) \\
& =\int_{\mathbb{R}^{d}} e^{-8 \pi^{2} t|\xi|^{2}} \lambda(d \xi) \\
& \leqslant \sup _{\xi \in \mathbb{R}^{d}}\left(e^{-8 \pi^{2} t|\xi|^{2}}\left(1+|\xi|^{2}\right)^{\eta}\right) \times \int_{\mathbb{R}^{d}} \frac{1}{\left(1+|\xi|^{2}\right)^{\eta}} \lambda(d \xi) \\
& \leqslant K_{\eta}\left(1 \vee\left(\frac{\eta}{8 \pi^{2}}\right)^{\eta} t^{-\eta}\right) .
\end{aligned}
$$

Based on the above inequality, it is easily to obtain (4.54). The proof is complete.

Acknowledgments: S. Shang is supported by the Fundamental Research Funds for the Central Universities (WK0010000057), Project funded by China Postdoctoral Science Foundation (2019M652174). R. Wang is supported by National Natural Science Foundation of China (11871382, 11671076, 11431014).

\section{REFERENCES}

[1] Bakry, D., Gentil, I., Ledoux, M.: Analysis and geometry of Markov diffusion operators. Grundlehren der Mathematischen Wissenschaften, 348. Springer, Cham, 2014.

[2] Bao, J., Wang, F.-Y., Yuan, C.: Transportation cost inequalities for neutral functional stochastic equations. Z. Anal. Anwend., 32(4), 457-475 (2013).

[3] van den Berg, M.: Gaussian bounds for the Dirichlet heat kernel. J. Funct. Anal., 88(2), 267-278 (1990).

[4] Boufoussi, B., Hajji, S.: Transportation inequalities for stochastic heat equations. Statist. Probab. Lett., 139, 75-83 (2018).

[5] Budhiraja, A., Dupuis, P.: A variational representation for positive functionals of infinite dimensional Brownian motion. Probab. Math. Statist., 20, 39-61 (2000). 
[6] Da Prato, G., Zabczyk, J.: Stochastic equations in infinite dimensions. Encyclopedia of Mathematics and its Applications, 152. Cambridge University Press, Cambridge, 1992.

[7] Dalang, R. C.: Extending the martingale measure stochastic integral with applications to spatially homogeneous s.p.d.e's. Electron. J. Probab., 4, 1-29 (1999).

[8] Dalang, R. C., Quer-Sardanyons, L.: Stochastic integrals for spde's: A comparison. Expo. Math., 29(1), 67-109 (2011).

[9] Djellout, H., Guillin, A., Wu, L.: Transportation cost-information inequalities and applications to random dynamical systems and diffusions. Ann. Probab., 32, 2702-2732 (2004).

[10] Dubhashi, D. P., Panconesi, A.: Concentration of measure for the analysis of randomized algorithms. Cambridge University Press, Cambridge, 2009.

[11] Feyel, D., Üstünel, A. S.: Monge-Kantorovitch measure transportation and Monge-Ampère equation on Wiener space. Probab. Theory Related Fields, 128(3), 347-385 (2004).

[12] Foondun, M., Khoshnevisan, D.: On the stochastic heat equation with spatially-colored random forcing. Trans. Amer. Math. Soc., 365, 409-458 (2013).

[13] Gozlan, N.: Transport inequalities and concentration of measure. ESAIM Proc. Surveys, 51, 1-23 (2015).

[14] Márquez-Carreras, D., Sarrà, M.: Large deviation principle for a stochastic heat equation with spatially correlated noise. Electron. J. Probab., 8, 1-39 (2003).

[15] Khoshnevisan, D.: Analysis of stochastic partial differential equations. CBMS Regional Conference Series in Mathematics, 119. American Mathematical Society, Providence, RI, 2014.

[16] Khoshnevisan, D., Sarantsev, A.: Talagrand concentration inequalities for stochastic partial differential equations. Stoch PDE: Anal. Comp. 1-20, https://doi.org/10.1007/s40072-019-00136-8, (2019).

[17] Lacker, D.: Liquidity, risk measures, and concentration of measure. Math. Oper. Res., 43(3), 813-837 (2018).

[18] Ledoux, M.: The concentration of measure phenomenon. Mathematical Surveys and Monographs, 89. American Mathematical Society, Providence, RI, 2001.

[19] Li, Y., Wang, R., Zhang, S.: Moderate deviations for a stochastic heat equation with spatially correlated noise. Acta Appl. Math., 139, 59-80 (2015).

[20] Massart, P.: Concentration inequalities and model selection. Lecture Notes in Mathematics, 1896. Springer, Berlin, 2007.

[21] Riedel, S.: Transportation-cost inequalities for diffusions driven by Gaussian processes. Electron. J. Probab., 22, 1-26 (2017).

[22] Saussereau, B.: Transportation inequalities for stochastic differential equations driven by a fractional Brownian motion. Bernoulli, 18(1), 1-23 (2012).

[23] Shang, S., Zhang, T.: Quadratic transportation cost inequalities under uniform distance for stochastic equations driven by multiplicative space-time white noise. ArXiv: 1906.07543v1 (2019).

[24] Talagrand, M.: Transportation cost for Gaussian and other product measures. Geom. Funct. Anal., 6(3), 587-600 (1996).

[25] Villani, C.: Optimal Transport: Old and New. Grundlehren der Mathematischen Wissenschaften, 338. Springer, Berlin, 2009.

[26] Walsh, J.: An introduction to stochastic partial differential equations. Lecture Notes in Mathematics, 1180. Springer, Berlin, 1986.

[27] Wang, F.-Y., Zhang, T.: Talagrand inequality on free path space and application to stochastic reaction diffusion. ArXiv: 1906.07543v1 (2019).

[28] Wu, L., Zhang, Z.: Talagrand's $T_{2}$-transportation inequality w.r.t. a uniform metric for diffusions. Acta Math. Appl. Sin. Engl. Ser., 20(3), 357-364 (2004).

[29] Wu, L., Zhang, Z.: Talagrand's $T_{2}$-transportation inequality and log-Sobolev inequality for dissipative SPDEs and applications to reaction-diffusion equations. Chinese Ann. Math. Ser. B, 27(3), 243-262 (2006). 
Current address, Shang, S.: School of Mathematics, University of Science and Technology of China, Hefei, 230026, China.

E-mail address: sjshang@ustc.edu.cn

Current address, Wang, R.: School of Mathematics and Statistics, Wuhan University, Wuhan, 430072, China

E-mail address: rwang@whu.edu.cn 